\title{
Current and Future Molecular Mechanism in Inflammation and Arthritis
}

\author{
Vikash Sharma ${ }^{1}$, Raj Kumar Tiwari ${ }^{1}$, Shiv Shankar Shukla ${ }^{1}$, Ravindra Kumar Pan- \\ $\operatorname{dey}^{1 *}$
}

'Columbia Institute of Pharmacy, Raipur, C.G., India

\section{Key Words}

inflammation, arthritis, cytokines, receptors, transcription factor

\author{
Abbreviations \\ IL- Interleukins \\ INF- Interferons \\ TNF- Tumor necrosis factors \\ JAK- Jannus Kinase \\ TGF- Transforming growth factor \\ proteins \\ NF-KB- Nuclear factor kappa Beta \\ COX- Cyclooxygenase \\ WBC- White blood cells \\ PDGF- Platelet derived growth factor \\ EGF- Epidermal growth factor \\ PUFAs- Polyunsaturated fatty acids \\ LOs - Lipoxygenases \\ TLR- Toll like receptor \\ GDP- Guanine diphosphate \\ GTP- Guanine triphosphate \\ PI3K - Phosphatidylinositol-3-Kinase \\ PLA2- Phospholipase A2
}

NSAIDs- Non steroidal Anti-inflammatory drugs

DMARDs- Disease modifying Antirheumatic drugs

STAT- Signal transducer and activator of transcription

DSAMPs- Damage- associated molecules patterns

\begin{abstract}
Inflammation is an immune response of the human body but excessive inflammation is taken as a major factor in the development of many diseases including autoimmune disorders, cancer and nerve disorders etc. In this regards the need is to suppress the inflammatory response. Suppression of extra or imperfect inflammatory response is not a big deal provided there is an exact knowledge of particular target in the body. Recent advancements in Pharmacological aspect made the therapy with improved outcomes in number of patients. Anticytokine therapy might be one of the important and novel approaches for inflammation and Arthritis. This can be achieved only when we go through the pathophysiology of expression and identification of mediators. Let's take an example of cytokine like interleukins (IL), chemokines, interferons (INF), tumor necrosis factors (TNF- $\alpha$ ), growth factors, and colony stimulating factors) release pathway which is a major signalling protein in inflammatory response. In the present study we have reviewed the recent pharmacological therapeutic advancement, inflammatory mediators, receptors, and major signalling pathways. Such information will not only provide the idea about the mechanism of action of Pharmaceuticals and molecular targets but also it provides a new aspect for drug designing and new corrective approaches in existing clinical medicines. This study will be a source of good information for the researchers working in the area of drug designing and molecular Pharmacology especially in anti-inflammatory and anti arthritic medicines for target based therapy.
\end{abstract}




\section{Introduction}

Inflammation is an imperative and fundamental response of the immune system against any harmful stimuli, microbial invasion, infected cells, toxic substances and injury, and acts by removal of injurious stimulus thereby initiating the healing process. Basically, it is the defense system of body. When inflammation goes significant, it develops serious complication including neurodegenerative disorders, autoimmune diseases, neoplastic diseases, and cardiac complications. Initially, inflammation commences as an acute condition and if failed to regulate the pro-inflammatory stimulus for prolonged period, it gets transformed into chronic form. Acute inflammation is regulated and chronic inflammation is deregulated form. But it's a challenge to differentiate between acute and chronic inflammation based on the molecular pharmacological basis [1]. The excess of the responses may be frightful in Rheumatoid Arthritis, Crohn's disease, inflammatory bowel disease, metabolic recently, management of Arthritis was a major challenge for the clinicians, the reason being delayed recognition, lack of knowledge about the physiological and molecular aspects. There are a limited number of drugs available like NSAIDs, which gives symptomatic relief from pain and inflammation but also show disappointing results and the side-effects, and lack the targeted therapy, which efficiently acts on selective targets and hit the exact cause. Recently, the research suggested that therapeutic relief can be given to the patient. In the past few years advances of new drugs like IL-6 Inhibitor, JAK inhibitor, anti TNF and currently available disease modifying anti rheumatic drugs (DMARDs) have provided relief in this context. Molecular Pharmacological understanding and efforts towards the signal transduction pathways of anti-inflammatory targets and their expression pattern is the major pathway to have an insight which serves as a tool for designing oral drug therapy pertaining to the enhancement of clinical use of natural and synthetic medicines [3].

\subsection{Recently developed treatment recom- mendations}

There has been tremendous development in drugs used for inflammatory ailments developed in recent years. DMARDs are the drugs indicated for the treatment of Rheumatoid Arthritis. These drugs have been designed in order to slow down the rate of deformity to the joints. Currently available drugs include NSAIDs and DMARDs from chemical origin or of biological origin i.e. tumor necrosis factor inhibitors, interleukin-6-inhibitors, and B-cell disrupting drugs [4].

\subsubsection{Conventional or Traditional Synthetic DMARDs}

Prevention of RA patient from the deformity and narrowing of joints can be achieved by using early diagnosis and treatment with DMARDs (Table 1). The prominent drugs modifying the disease condition from synthetic origin includes the analog of folic acid (Methotrexate), Pyrimidine synthesis inhibitor (Leflunomide) and Immunosuppressant (Sulfasalazine) [5].

\subsubsection{Novel DMARDs or Target based therapy}

\subsubsection{Biologics}

The mechanism of action of biological DMARDs (Table 2) is very much selective as they act by interfering with the production of cytokines, signal transduction for initiation of T cells thereby reducing the number of B cells. Biological DMARDs include TNF- $\alpha$ inhibitor (Infliximab), T cell and B cell-targeted therapy (Abatacept, Rituximab respectively) and cytokines targeted therapy (Tocilizumab) [6].

Table 1 Drugs used for arthritis. Conventional Disease Modifying Anti-Rheumatic Drugs (DMARDs)

\begin{tabular}{|l|l|}
\hline \multicolumn{1}{|c|}{ Conventional DMARDs } & \multicolumn{1}{c|}{ Mechanism of Action } \\
\hline Methotrexate & Folic acid antagonist and Increase Adenosine release \\
\hline Sulfasalazine & $\begin{array}{l}\text { Inhibition of Inflammatory mediators by Arachidonic Acid } \\
\text { Inhibition }\end{array}$ \\
\hline Hydroxychloroquine & $\begin{array}{l}\text { Increases lysosomal pH in antigen-presenting cells, Blockade of } \\
\text { Toll like receptor signaling }\end{array}$ \\
\hline Leflunomide & Inhibition of dihydroorotate dehydrogenase (DHODH), an \\
& enzyme having role in pyrimidine synthesis \\
\hline
\end{tabular}


Table 2 Drugs used for arthritis. Conventional Disease Modifying Anti-Rheumatic Drugs (DMARDs)

\begin{tabular}{|l|l|}
\hline \multicolumn{1}{|c|}{ Biologics DMARDs } & \multicolumn{1}{c|}{ Mechanism of Action } \\
\hline Infliximab & Prevent Binding of TNF- $\alpha$ to its receptor \\
\hline Etanercept & Prevent Binding of TNF- $\alpha$ and TNF- $\beta$ to its receptor \\
\hline Adalimumab & Prevent Binding of TNF- $\alpha$ to its receptor \\
\hline Abatacept & Inhibition of T-lymphocytes, preventing cell mediated immune \\
& response \\
\hline Tocilizumab & IL-6 Inhibition \\
\hline Tofacitinib & Inhibits Janus kinase (JAK) 1 and JAK3 \\
\hline
\end{tabular}

\section{Molecular Pharmacology in Rheumatoid Arthritis}

\subsection{Cytokines}

Cytokines are the major secreted and signalling proteins as a response against inflammation and the immune system. Cytokines bind to their receptors present over the membrane of the target cell, which initiates the signal transduction cascade resulting in altered gene expression in the target cell [7]. Cytokines include lymphokine, monokine, chemokine, and interleukin. Generally, cytokines show three types of actions. Cytokine can either bind to receptors on the membrane of the same cell (autocrine), or nearby cells (paracrine) and cells in the distal part of the human body. Cytokine binds with the receptor of the target cells generally activate the receptors and secretion of other cytokines. Cytokines are either pro-inflammatory or anti-inflammatory cytokines [8]. Pro-inflammatory mediators include IL-1, IL-6, transforming growth factor $\beta$ (TGF $\beta$ ), interferon $\gamma$ (IFN $\gamma$ ) and Tumor necrosis factor $\alpha$ (TNFa), other cytokines include IL-4, IL-12, IL-10 [9].

Cytokines have diverse physiological roles which are inclusive of the development of cell-mediated and humoral immunity, inflammatory reactions, regulation of cell proliferation, wound healing. Activities of some most common cytokines have been summarized in Table 1 [10].

\subsection{Transcription factors involved in Arthritis}

\subsubsection{Nuclear factor NF-KB}

There is a fundamental role of transcription factors in maintaining the expression of pro-inflammatory mediators in the progress of inflammation. These factors include mainly NF-KB, STAT1/STAT3. NF-KB expresses many complexes of members of the Rel (Reticuloendotheliosis) protein family [11]. Mainly this protein consists of two subunits p50 and p65 commonly referred to NF-KB/Rel complex. Initially, this remains in an inactive state in the cytoplasm of resting cells in an association of inhibitory IKB family (composed of two kinase subunits, IKBa and IKB $\beta$ ) [12]. Activation of this protein is achieved by pro-inflammatory catalyst as cytokines, Lipopolysaccharide (LPS) by proteasomal degradation. Following the activation, the next step is the displacement of attachment of NF-KB to the promoter region of genes, results in activation of pro-inflammatory mediators, such as COX-2 and iNOS, etc [13]. NF-KB signaling causes the degradation of IkB results in the migration of NF- $\mathrm{KB}$ inside the nucleus where it serves as transcription factor. Finally, this contributes to the inflammatory responses in the cell. This was the first identified transcription factor in B cell having a significant role in inflammatory responses and other cellular responses as apoptosis. NF-KB is also supposed to have a significant role play in diseases such as arthritis, carcinoma, asthma, etc [14].

\subsubsection{STATs}

STATs transcription factors are also of immense importance in inflammation and many other immunological responses. STATs are responsible for sequential signaling initiated by cytokines; IL- 6 family, epidermal growth factor (EGF) and platelet-derived growth factor (PDGF) [15]. Activation of STATs initiated via cytokine receptor during phosphorylation of tyrosine kinase, JAK family. JAK-STAT pathway is involved in response to several cytokines and their response is also different as well. Initially, binding of cytokines to these receptors induces the dimerization of these cytokine receptors followed by attachment of a tyrosine kinase unit (JAK to associate) and thus, phosphorylation of receptor dimer takes place. JAK represents a group 


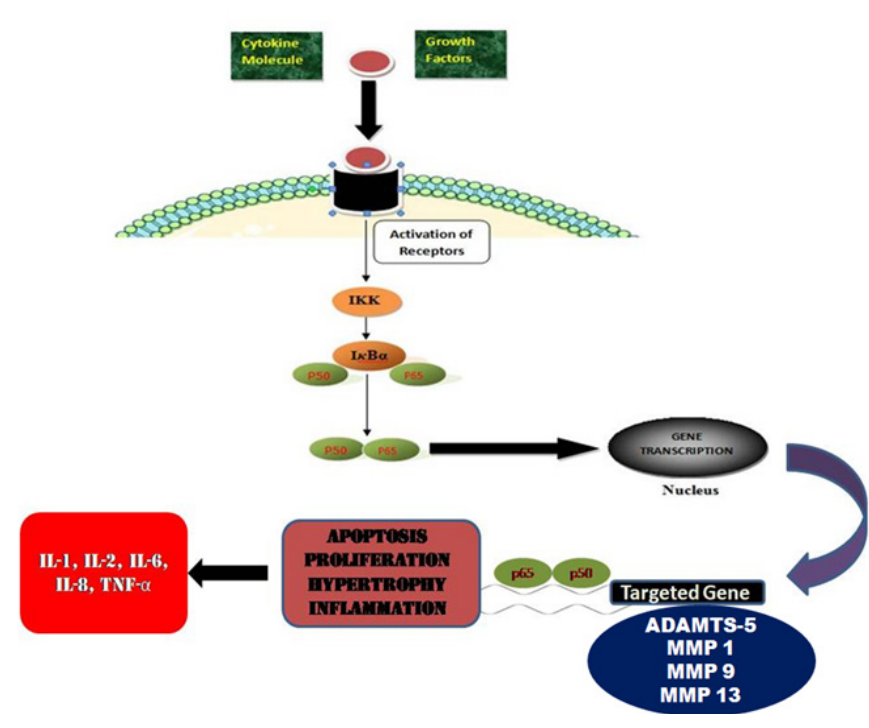

Figure 1 Nuclear Factor-кB pathway. The nuclear factor-kappa (NF-_B) level differently regulates cartilage homeostasis. Upon ligand binding to the receptor present on the cell surface, the activated IKK causes phosphorylation of IKB which further results in NF-KB activation which translocate to the nucleus where serves as a transcription factor and bind to DNA to induce NF-KB target gene transcription which further results in various cellular responses like Apoptosis, Inflammation, Proliferation and Hypertrophy in chondrocytes. NF- $\mathrm{KB}$ induces catabolic gene expression through NF-_B response elements located in the promoters of the MMP1, MMP9, MMP13 and ADAMTS5 genes. of protein molecule in which each protein has specificity for a unique receptor [16]. STATs are similarly a group of transcription factors, subject to phosphorylation by JAK. This phosphorylation of STAT stabilizes the Src homology 2 (SH2) domains by intercommunication of phosphotyrosine and SH2. After that, there is dimerization of STAT, reaching to nucleus and thus results in activation of a gene responsible for inflammatory modulators and other proteins regulating cell proliferation [17].

Currently, seven members of STATs family were identified, among them only five (STAT1-4 STAT6), execute as transcription factors. STAT1 and STAT2 were identified as mediators of IFN signaling. STAT2 involved in IFN type I (IFN- $\alpha, \beta$ ) signaling, whereas STAT1 mediates signaling of both type I as well as type II IFN (IFN-Y). These two types of IFN are responsible for the inflammatory response in viral infection and bacterial defense. STAT 3 mediates IL-6 and IL-10. STAT 4 and STAT 6 have a significant involvement in acquired immune responses. Activation of STAT 4 depends on IL-12 and STAT 6 on IL-4, IL-13. STAT 4 play a major role in the development of autoimmune disorders whereas STAT6 is involved in allergic inflammatory reactions [18].

\subsubsection{Toll like Receptors}

Toll genes were first identified in the1980s and the first toll like receptor (TLR) was identified in 1996 [19]. There are approximately 13 TLRs and out of them, 11 are present in the human body. TLR functions as a microbial signature and are referred to as pathogen-associated molecular patterns (PAMPs). The molecules produced by damaged

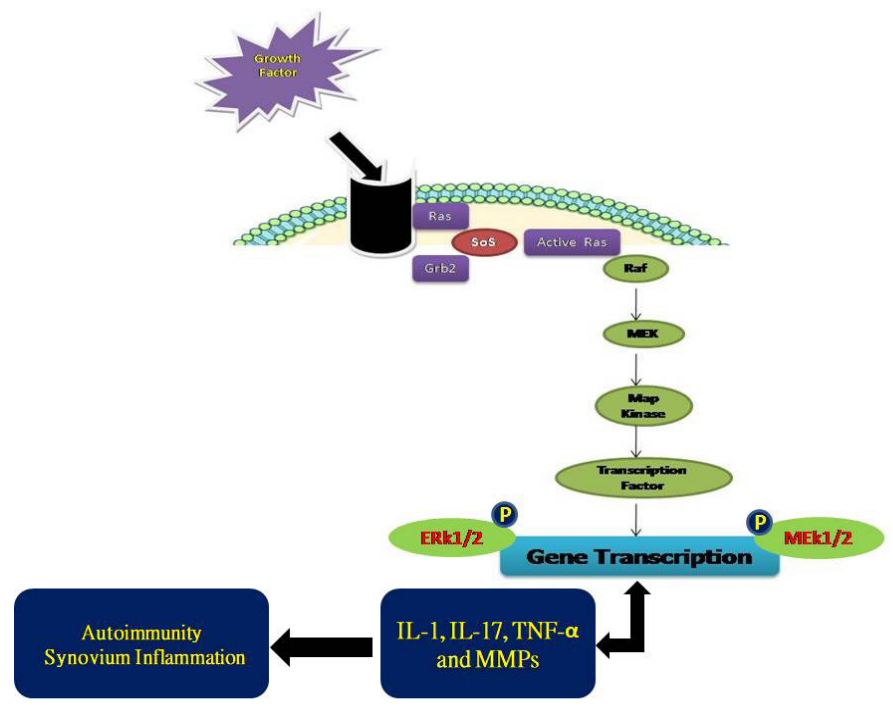

Figure 2 Mitogen-Activated Protein Kinase (MAPK) cascade. This pathway plays a crucial role $\mathrm{n}$ the progression of Rheumatoid arthritis. MAPK pathway (right) are activated by the pro-inflammatory cytokines TNF- $\alpha$ and IL-1. MAPK cascade initiated by binding of ligands such as growth factors mitogens, cytokines and stress signals. MAPK cascade after phosphorylation of its components serves as transcription factor and binds to the DNA for genes coding for various cytokines including TNF- $\alpha$ and IL-1, other inflammatory molecules, such as prostaglandins (PGs), and proteases, such as the matrix metalloproteinases (MMPs). ERK1/2 controls PGE2 and MMP-1 production which contributes to the inflammation, angiogenesis, recruitment of cells from both innate and adaptive arms of the immune system, cartilage damage and bone erosion. 
cells, are referred to as damage-associated molecules patterns (DAMPs) [20]. The initial activation is done by some specific encoded pattern- recognition receptors (PRRs) provide by the innate immune response. PRRs activate the signaling pathways which further initiates the innate immune response through the synthesis of cytokines and many other mediators. TLRs are expressed in inborn immune cells like macrophages. TLRs are grouped into two families according to their position across the cell; cell surface (Cell surface TLRs include TLR1, TLR2, TLR4-6, and TLR10) TLRs and intracellular TLRs (the endosome and include TLR3, TLR7, TLR8, TLR9, TLR11, TLR12, and TLR13) [21, 22].

\section{Pathways in Inflammatory Signaling:}

\subsection{Nuclear Factor-kB pathway}

In the inactive stage, NF- $\mathrm{KB}$ complexes are attached with IKB kinase- $\alpha$ (IKK $\alpha$ ), IKB kinase- $\beta$ (IKK $\beta$ ) and IKK $\gamma$ / NEMO in the cytoplasm. This complex is activated by attachment of ligands as cytokine, growth factors or damaged microbial cells to the receptors present on the cell surface. The activated IKK complex causes phosphorylation of IKB. Further, there is proteasomal degradation of Phosphorylated IKB and causes the NF-KB activation, which reaches in the nucleus where serves as a transcrip- tion factor for theexpression of specific target genes (Fig. 1) [23].

\subsection{Mitogen-Activated Protein Kinase}

\section{(MAPK) cascade}

Ras is a product of proto-oncogene, works similar to G-protein. It transmits the signals through (GDP/GTP) SH2 domain protein Grb, where phosphorylation occurs by tyrosine receptor kinase. This causes Ras activation which results in the activation of Raf. In the next step, there is phosphorylation of both Ras and Raf. At last, there is the phosphorylation of various transcription factors by MAP kinase, interfere in transcription in the nucleus [24]. (Fig. 2)

\subsection{JAK-STAT signaling pathway}

Binding of cytokines to the receptors induces dimerization of these receptors and causes the phosphorylation of JAKs and STATs. Activation of STAT causes dimerization. These dimers serve as a transcription factor and causes gene transcription in the nucleus [25, 26] (Fig. 3)

\subsection{Phosphatidylinositol-3-Kinase (PI3K)/}

\section{Akt signaling pathway}

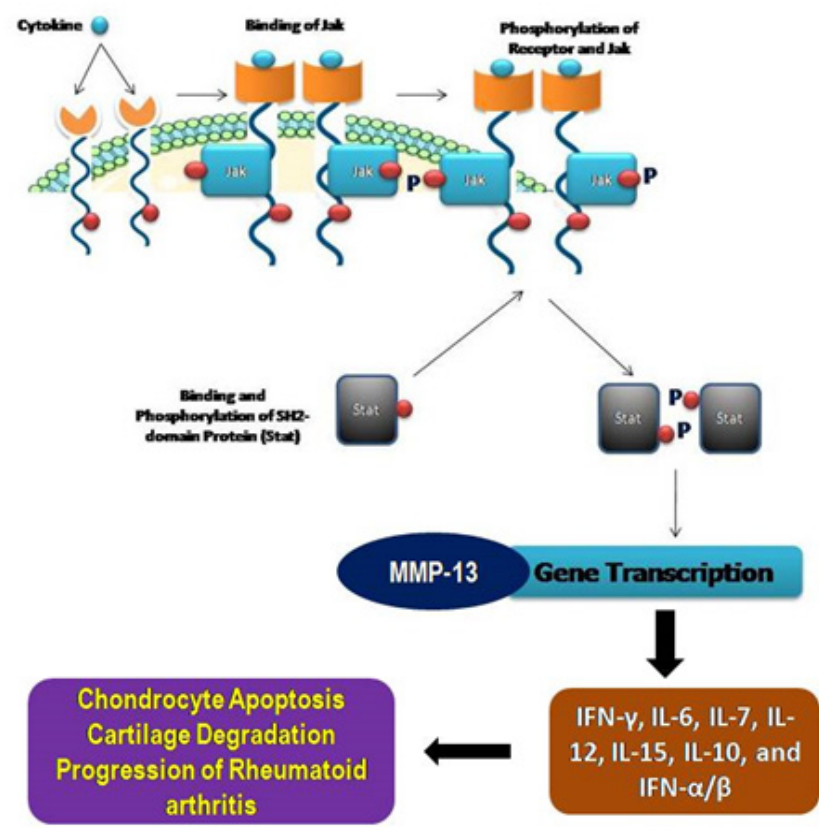

Figure 3 JAK-STAT signaling pathway. In mammals, the JAK/STAT pathway is the principal signaling mechanism for a wide array of cytokines and growth factors. Upon ligand binding, Jak associated with cytokine receptors undergo transphosphorylation and mediate phosphorylation of specific tyrosine residues on the cytokine receptor. Binding of cytokines to the receptors induces dimerization of these receptors and causes the phosphorylation of JAKs and STATs. Activation of STAT causes dimerization. These dimers serve as a transcription factor and causes transcription of Matrix Metallopeptidase 13(MMP-13). The JAK/STAT signal transduction pathway is utilized by many cytokines and growth factors that regulate gene expression and cellular activation, proliferation, and differentiation. 
PI3K/Akt Pathway is one of the most important pathways which play a pivotal role in modulating cellular growth, proliferation, metabolism, and angiogenesis. Akt is a serine/threonine kinase consists of a kinase domain and a pleckstrin homology, initially identified as proto-oncogene. Binding of ligand to the receptor causes activation of PIP3 results in further signaling. Activation of PIP3 further activates Akt and PKB. Akt induces activation of nuclear factor NF-KB which has a significant role in inflammation [27].

\subsection{Arachidonic Acid Pathway}

The arachidonic acid pathway is one of the most significant pathways which release several inflammatory mediators as Prostaglandins, thromboxanes, and leukotrienes. Activated Phospholipase A2 (PLA2) causes the formation of Arachidonic Acid from the cell membrane phospholipid [28-29]. Thus, Arachidonic acid is metabolized in to different mediators by some enzymatic reactions. Action of cyclooxygenase enzyme (COX) form prostaglandins and thromboxanes from Arachidonic acid. Similarly, action of 5-lipoxygenase (5-LOX) form leukotrienes [30, 31] (Fig. 4). Eicosanoids is the term coined from Greek word 'Eikosi' means twenty, includes one of a family of 20 locally acting biologically active signal molecules, formed by enzymatic reactions followed by arachidonic acid and polyunsaturated fatty acids (PUFAs) oxidation. As a result of these reactions are prostaglandins (PG), thromboxane (TXA), leukotrienes (LT), endocannabinoids and isoeicosanoids
[32]. Eicosanoids play a crucial role during immunological responses. Eicosanoids formed by three different pathways involving three different types of enzymes: cyclooxygenases (COXs), epoxygenases, and lipoxygenases (LOs). COX enzyme is physiologically very important enzyme as it is involved in the synthesis of Prostaglandins (PG), which is a very significant inflammatory mediator [33]. Synthesis of Eicosanoids is initiated by starting enzyme Phospholipase A2 (PLA2), which triggers the release of Arachidonic acid from membrane phospholipids. There are various enzymatic reactions for the metabolism of Arachidonic acid [34].

\subsubsection{Cyclo-oxygenase (COX)}

COX is essentially found in two forms COX-1and COX2. The metabolism of Arachidonic acid produces Prostaglandin $\mathrm{H} 2$ (PGH2), and formation of many other isomeric forms of prostanoids in different region of the body which depends on the cell type and degree of cellular activation [35] as PGD2, PGE2, PGF2 $\alpha$, thromboxane A2 (TXA2), PGI2 are found in vascular system, smooth muscle, platelets, and gastric mucosa respectively. PGE2 further activates PGE2 receptor which ultimately demonstrates the signs of inflammation and pain. PGE2 and TXA2 are found to be more significant in inflammatory reactions [36]. PGE2 produces its effect by another pathway; MAPK/ERK $[37]$.

\subsubsection{Lipo-oxygenase (LOX)}

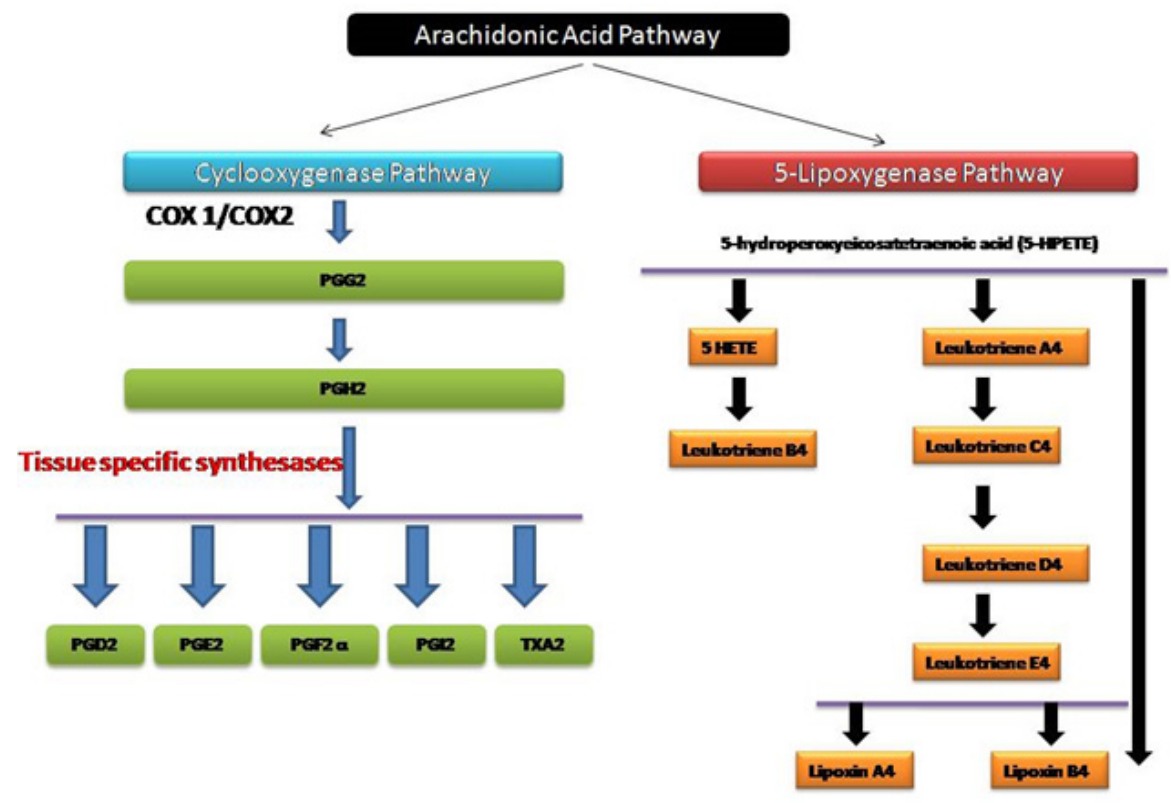

Figure 4 Arachidonic Acid Pathway. This Pathway involves the release of inflammatory mediators as Prostaglandins, thromboxanes, and leukotrienes as depicted in the figure. Phospholipase A2 activation initiates this pathway results in formation of Arachidonic Acid from the cell membrane. Then Arachidonic acid is metabolized into different mediators by some enzymatic reactions in presence of cyclooxygenase enzyme (COX) and Lipoxygenase (LOX). 
This pathway is mainly responsible for the production of leukotrienes and lipoxins. One significant role of this pathway is respiratory tract inflammation by enhancing bronchial constriction. Leukotrienes are produced by leukocyte like LTC4 production as a response to inflammation and cell injury. Similar to TXA2, it is a strong vasoconstrictor and acts via the Gq-protein pathway [38]. Leukotrienes and prostaglandins may also promote edema by making the vascular endothelium more "leaky" $[39,40]$.

\section{Conclusion}

Inflammation plays an essential role in the progression and development of number of ailments as mentioned in the study. This review largely focuses on the inflammatory mediators, their role, release pathways and physiological significance. In this review, we have tried to summarize these mediators as molecular targets in the therapy of inflammation and designing of novel medicines. Now-adays, new drug designing approaches have trended for targeted drug delivery system. As Arachidonic acid pathway is the specific target for the most prominent anti-inflammatory agents viz. Non-steroidal Anti-inflammatory drugs (NSAIDs). NSAIDs affect the arachidonic acid pathway and provide only symptomatic relief in case of arthritis and inflammation. Likewise, there are several other signaling molecules and transcription factors that have been summarized in the study may prove significant for relieving and targeting approach in the treatment of inflammation and Arthritis. This study might also be helpful in determination of possible method of such existing drugs whose mechanism is still unknown, in case of Ayurvedic and herbal medicines for the development of new drugs. This study possibly can be a breakthrough in herbal drug research for inflammatory ailments. Thus, to conclude that if a drug is showing anti-inflammatory action, it might be inhibiting one or many of the above mentioned targets. This study therefore provides the potential molecular aspect to young researchers working in drug development for inflammation and arthritis.

\section{Conflict of linterest}

The authors have no conflict of interest.

\section{ORCID}

Vikas Sharma. http://orcid.org/0000-0001-6396-7583

Raj Kumar Tiwari. http://orcid.org/0000-0001-7373-4679

Shiv Shankar Shukla. http://orcid.org/0000-0001-5291-577X Ravindra Kumar Pandey. http://orcid.org/0000-0003-4469238X

\section{References}

1. Chen L, Deng H, Cui H, Fang J, Zuo Z, Deng J, et al. Inflammatory Responses and Inflammation-Associated Diseases in Organs. Oncotarget. 2018;9(6):7204-7218.

2. Korniluk A, Koper O, Kemona H, Dymicka-Piekar- ska D. From inflammation to cancer. Ir J Med Sci. 2017;186(1):57-62.

3. Gavrilă BI, Ciofu C, Stoica V. Biomarkers in Rheumatoid Arthritis, what is new? J Med Life. 2016;9(2):144148.

4. Burmester GR, Pope JE. Novel Treatment Strategies in Rheumatoid Arthritis. Lancet. 2017;389(10086):23382348.

5. Guo Q, Wang Y, Xu D, Nossent J, Pavlos NJ, Xu J. Rheumatoid Arthritis: Pathological Mechanisms and Modern Pharmacologic Therapies. Bone Res. 2018;6(15):114.

6. Brown PM, Pratt AG, Isaacs JD. Mechanism of Action of Methotrexate in Rheumatoid Arthritis, and the Search for Biomarkers. Nat Rev Rheumatol. 2016;12(12):731742.

7. Tasneem S, Liu B, Li B, Choudhary MI, Wang W. Molecular Pharmacology of Inflammation: Medicinal Plants as Anti-Inflammatory Agents. Pharmacol Res. 2019;139(2019):126-140.

8. Zhang JM, An J. Cytokines, Inflammation and Pain. Int Anesthesiol Clin. 2007;45(2):27-37

9. Turner MD, Nedjai B, Hurst T, Pennington DJ. Cytokines and Chemokines: At the Crossroads of Cell Signalling and Inflammatory Disease. Biochim Biophys Acta. 2014;1843(11):2563-2582.

10. Kindt TJ, Goldsby RA, Osborne BA. Kuby Immunology. 6th ed. New York: Freeman press; 2007.

11. Hammaker D, Sweeney S, Firestein G. Signal transduction networks in rheumatoid arthritis. Ann Rheum Dis. 2003;62(Suppl 2):ii86-ii89.

12. Seetharaman R, Mora AL, Nabozny G, Boothby M, Chen J. Essential Role of T Cell NF-kappa B Activation in Collagen-Induced Arthritis. J Immunol. 1999;163(3):157783.

13. Wu Y, Antony S, Meitzler JL, Doroshow JH. Molecular mechanisms underlying chronic inflammation-associated cancers. Cancer Lett. 2014;345(2):164-173.

14. Fitzgerald KA, Palsson-McDermott EM, Bowie AG, Jefferies CA, Mansell AS, Brady G, et al. Mal (MyD88adapter-like) is Required for Toll-like receptor-4 Signal Transduction. Nature. 2001;413(6851):78-83.

15. Kaplan MH. STAT signaling in inflammation. JAK-STAT. 2013;2(1);1-3.

16. Yu H, Pardoll D, Jove R. STATs in Cancer Inflammation and Immunity: A Leading Role for STAT3. Nat Rev Cancer. 2009;9(11):798-809.

17. Rawlings JS, Rosler KM, Harrison DA. The JAK/STAT signaling pathway. J Cell Sci. 2004;117(8):1281-1283.

18. Seif F, Khosmirsafa M, Aazami H, Mohsenzadegan M, Sedighi G, Bahar M. The Role of JAK-STAT Signaling Pathway and Its Regulators in the Fate of T Helper Cells. Cell Commun Signal. 2017;15(1):23.

19. Kawasaki T, Kawai T. Toll-Like Receptor Signaling Pathways. Front Immunol. 2014;5(461):1-8.

20. Takeda K, Akira S. Toll-like Receptors. Curr Protoc Immunol. 2015;109:14.12.1-14.12.10.

21. Takeda K, Kaisho T, Akira S. Toll-like Receptors. Annu Rev Immunol. 2003;21:335-76.

22. Kaisho T, Akira S. Toll-like Receptor Function and Signaling. J Allergy Clin Immunol. 2006;117(5):979-87. 
23. Liu T, Zhang L, Joo D, Sun SC. NF-KB Signaling in Inflammation. Signal Transduct Target Ther. 2017;2:17023.

24. Soares-Silva M, Diniz FF, Gomes GN, Bahia D. The Mitogen-Activated Protein Kinase (MAPK) Pathway: Role in Immune Evasion by Trypanosomatids. Front Microbiol. 2016;7(183):1-9.

25. Rawlings JS, Rosler KM, Harrison DA. The JAK/STAT signaling pathway. J Cell Sci. 2004;117(8):1281-1283.

26. Harrison DA. The JAK/STAT Pathway. Cold Spring Harb Perspect Biol. 2012;4(3):a011205.

27. Liu P, Cheng H, Roberts TM, Zhao JJ. Targeting the Phosphoinositide 3-kinase Pathway in Cancer. Nat Rev Drug Discov. 2009;8(8):627-44.

28. Samuelsson B. Arachidonic Acid Metabolism: Role in Inflammation. Z Rheumatol. 1991;50(Suppl 1):3-6.

29. Yang G, Chen L. An Update of Microsomal Prostaglandin E Synthase-1 and PGE2 Receptors in Cardiovascular Health and Diseases. Oxid Med Cell Longev. 2016;2016:5249086.

30. Tallima H, Ridi RE. Arachidonic Acid: Physiological Roles and Potential Health Benefits -A Review. J Adv Res. 2017;11:33-41.

31. Hanna VS, Hafez EAA. Synopsis of arachidonic acid metabolism: A review. J Adv Res. 2018;11:23-32.

32. Poorani R, Bhatt AN, Dwarakanath BS, Das UN. COX2, Aspirin and Metabolism of Arachidonic, Eicosapentaenoic and Docosahexaenoic Acids and Their Physiological and Clinical Significance. Eur J Pharmacol. 2016;15(785):116-132.

33. Khanapure SP, Garvey DS, Janero DR, Letts LG. Eicosanoids in Inflammation: Biosynthesis, Pharmacology, and Therapeutic Frontiers. Curr Top Med Chem. 2007;7(3):311-40.

34. Pannunzio A, Coluccia M. Cyclooxygenase-1 (COX-1) and COX-1 Inhibitors in Cancer: A Review of Oncology and Medicinal Chemistry Literature. Pharmaceuticals (Basel). 2018;11(4):101.

35. Dennis EA, Norris PC. Eicosanoid Storm in Infection and Inflammation. Nat Rev Immunol. 2015;15(8):51123.

36. Ricciotti E, FitzGerald GA. Prostaglandins and Inflammation. Arterioscler Thromb Vasc Biol. 2011;31(5):9861000.

37. Smith WL, DeWitt DL, Garavito RM. Cyclooxygenases: Structural, Cellular, and Molecular Biology. Annu Rev Biochem. 2000;69:145-82.

38. Requelme MA, Burra S, Kar R, Lampe P, Jiang JX. Mitogen-activated Protein Kinase(MAPK) Activated by Prostaglandin E2Phosphorylates Connexin 43 and Closes Osteocytic Hemichannels in Response to Continuous Flow Shear Stress. J Biol Chem. 2015;290(47):28321-8.

39. Paul CN, Dennis EA. A Lipidomic Perspective on Inflammatory Macrophage Eicosanoid Signaling. Adv Biol Regul. 2014;54:99-110.

40 Sirois P, Borgeat P. Pharmacology of the Leukotrienes. J Pharmacol. 1984;15(Suppl 1):53-68. 